\title{
Traveltime and amplitude calculation using a perturbation approach
}

\author{
Changsoo Shin*, Kurt J. Marfurt ${ }^{\ddagger}$, Kwon Gyu Park $\ddagger$, Dong-Joo Min**, \\ Kwangjin Yoon*, Dongwoo Yang*, Taeyoung Ha*, \\ Seungwon Ko*, Wonsik Kim*, and Soonduk Hong*
}

\begin{abstract}
Accurate amplitudes and correct traveltimes are critical factors that govern the quality of prestack migration images. Because we never know the correct velocity initially, recomputing traveltimes and amplitudes of updated velocity models can dominate the iterative prestack migration procedure. Most tomographic velocity updating techniques require the calculation of the change of traveltime due to local changes in velocity. For such locally updated velocity models, perturbation techniques can be a significantly more economic way of calculating traveltimes and amplitudes than recalculating the entire solutions from scratch.

In this paper, we implement an iterative Born perturbation theory applied to the damped wave equation algorithm. Our iterative Born perturbation algorithm yields stable solutions for models having velocity contrasts of $30 \%$ about the initial velocity estimate, which is significantly more economic than recalculating the entire solution.
\end{abstract}

\section{INTRODUCTION}

One of the most critical factors governing the quality of Kirchhoff-type prestack images is the accuracy of traveltimes and amplitudes from each source and receiver point to the subsurface. Traveltime computation and prestack migration are directly linked to the correctness of the velocity model. We almost always need to update the velocity model resulting in an iterative migration process. It is necessary to repeat the test-update cycle until some convergence criterion is satisfied. Unfortunately, this iterative approach can be quite timeconsuming; traveltime calculations may account for as much as $40 \%$ of the cost of Kirchhoff prestack depth migration (Zhu and Lines, 1998). Even when we are interested in modifying only a small target region, conventional algorithms require us to recalculate the entire traveltime tables from scratch. For this reason, we have developed perturbation techniques that will allow us to effectively deal with a suite of sequential velocity models.

Ettrich and Gajewski (1998) have used first-order perturbation theory to compute traveltimes corresponding to a slightly perturbed model by adding a correction term, $\Delta t$, obtained by simple integration of slowness differences along raypaths perpendicular to Vidale's (1998) traveltime wavefront generated on a reference medium. Vidale's method is based on the solution of the eikonal equation, which is in turn a highfrequency approximation to the scalar wave equation. Vidale's method is computationally efficient, and easy to extend to three dimensions. However, his method may encounter difficulties in treating complex models having a large velocity contrast, geometrical shadow zones, and local low-velocity zones where waves are trapped. Podvin and Lecomte (1991) improved the stability problem of the eikonal solver for high velocity contrast and shadow zones. Van Trier and Symes (1991) vectorized the eikonal solver, whereas Qin et al. (1992) improved the eikonal solver by using expanding wavefronts.

Shin et al. (2002) proposed a traveltime computation algorithm (called SWEET) that uses strongly damped wavefields in the Laplace-transformed domain. They first used the standard model of approximating the seismic wavefield by a reflectivity function consisting of spikes convolved with a simple source wavelet. Next, they solved the wave equation in the Laplacetransformed domain, where they could suppress all events after the first arrival automatically because the Laplace-transformed wavefield includes a strong damping factor $\left(e^{-s t}\right.$, where $s$ is the Laplace frequency and $t$ is the time). By solving the damped wave equation and calculating the derivative of the wavefield at an appropriately chosen Laplace frequency, they were able to calculate both the traveltime and amplitude of the first arrival.

Published on Geophysics Online June 24, 2002. Manuscript received by the Editor January 10, 2001; revised manuscript received March 26 , 2002. ${ }^{*}$ Seoul National University, School of Civil, Urban \& Geosystem Engineering, San 56-1, Sillim-dong, Kwanak-Ku, Seoul, 151-740, Korea. E-mail: css@model.snu.ac.kr; yhcha@gpl.snu.ac.kr.

†University of Houston, Allied Geophysical Laboratories, Department of Geoscience, Houston, Texas 77204-5006. E-mail: kmarfurt@uh.edu.

${ }_{* *}$ Korea Ocean Research \& Development Institute Ansan, Post Office Box 29, Kyungki, 425-600, Korea. E-mail: djmin@kordi.re.kr.

(C) 2002 Society of Exploration Geophysicists. All rights reserved. 
Marfurt and Shin (1989) used an iterative Born perturbation of the full wave equation to obtain the full waveform seismograms for a slightly perturbed target lying within a reference medium. In electromagnetic applications, Hohmann (1990) used the perturbation technique to simulate an induced polarization response.

In this paper, we apply Born perturbation theory to Shin et al.'s (2002) SWEET algorithm in order to efficiently compute traveltimes and amplitudes corresponding to a suite of related velocity models. We begin by calculating the unperturbed damped wavefields using a finite-element method. We then obtain perturbed damped wavefields by the successive application of iterative Born perturbation theory. We then estimate traveltimes and amplitudes of the first arrivals using the same technique derived by Shin et al. (2002).

\section{METHODOLOGY}

When we solve the wave equation by using the finite-element (or finite-difference) method, we obtain in general

$$
\mathbf{M u ̈}+\mathbf{K u}=\mathbf{f}
$$

where $\mathbf{M}$ is the mass matrix, $\mathbf{K}$ is the stiffness matrix, $\mathbf{f}$ is the source vector, and $\mathbf{u}$ is the solution (Zienkiewicz, 1977; Marfurt, 1984). In the Laplace domain, we can rewrite equation (1) as

$$
\mathbf{S U}=\mathbf{F},
$$

where $\mathbf{S}$ is the impedance matrix defined as

$$
\mathbf{S}=s^{2} \mathbf{M}+\mathbf{K}
$$

and $\mathbf{U}(s)$ and $\mathbf{F}(s)$ are the Laplace transforms of $\mathbf{u}(t)$ and $\mathbf{f}(t)$, defined as

$$
\mathbf{U}(s)=\int_{0}^{\infty} \mathbf{u}(t) e^{-s t} d t
$$

and

$$
\mathbf{F}(s)=\int_{0}^{\infty} \mathbf{f}(t) e^{-s t} d t .
$$

From equations (4) and (5), we note that we can regard the Laplace-transformed wavefield as the wavefield, $u$, damped by the factor $e^{-s t}$ and integrated over time (Shin et al., 2002). If we perturb the density and/or the bulk modulus of the medium, equation (2) becomes

$$
\left(\mathbf{S}_{0}+\delta \mathbf{S}\right)\left(\mathbf{U}_{0}+\delta \mathbf{U}\right)=\mathbf{F},
$$

where $\mathbf{S}_{0}$ and $\mathbf{U}_{0}$ are the impedance matrix and wavefields corresponding to the reference medium, and $\delta \mathbf{U}$ is a small change to the solution due to a small change in the impedance matrix, $\delta \mathbf{S}$. Expanding equation (6) using equation (2) and neglecting second-order terms, we obtain

$$
\mathbf{S}_{0} \delta \mathbf{U}=-(\delta \mathbf{S}) \mathbf{U} .
$$

Thus, the scattered wavefield is given by

$$
\delta \mathbf{U}=-\mathbf{S}_{0}^{-1}(\delta \mathbf{S}) \mathbf{U},
$$

and the perturbed wavefield is given by

$$
\mathbf{U}=\mathbf{U}_{0}+\delta \mathbf{U} \text {. }
$$

We note that our reference medium represented by $\mathbf{S}_{0}$ may be arbitrarily complex since we calculate $\mathbf{U}_{0}$ using a finiteelement method. The computation of the inverse matrix $\mathbf{S}_{0}^{-1}$ of equation (8), which is called a Green's operator (Stolt and Weglein, 1985; Tarantola, 1987), is in general quite expensive. However, we note that we have already calculated $\mathbf{S}_{0}^{-1}$ in order to obtain $\mathbf{U}_{0}$ in equation (2). In practice, we never formally calculate $\mathbf{S}_{0}^{-1}$ but rather factor $\mathbf{S}_{0}$ into sparse upper and lower triangular matrices. Thus, the perturbed wavefields can be obtained by simple matrix forward and backward substitutions, which need only a fraction of the computer time required to factor $\mathbf{S}_{0}$. For any perturbation, we form $\delta \mathbf{S}$ using a conventional finite-element assemblage process. If our perturbations are spatially limited, only a small number of elements will be needed to represent them, and thus $\delta \mathbf{S}$ becomes a small, sparse matrix that has only local numerical support.

If we approximate the wavefield within the perturbation $\mathbf{U}$ in equation (8) using the unperturbed wavefields $\mathbf{U}_{0}$, we obtain the first-order Born approximation (Stolt and Weglein, 1985; Tarantola, 1987),

$$
\mathbf{U}_{1}=\mathbf{U}_{0}-\mathbf{S}_{0}^{-1} \delta \mathbf{S} \mathbf{U}_{0} .
$$

If we successively update this wavefield in the perturbation for each iteration, we obtain the well-known iterative Born approximation, that is,

$$
\mathbf{U}_{i}=\mathbf{U}_{0}-\mathbf{S}_{0}^{-1} \delta \mathbf{S} \mathbf{U}_{i-1}, \quad \text { for } \quad i=1,2,3, \cdots
$$

The stronger the velocity perturbation above the reference velocity is, the higher the order of approximation is required to obtain an accurate solution.

\section{NUMERICAL EXAMPLES}

\section{Accuracy and convergence}

The accuracy of our traveltime computations using a perturbation technique is governed by the accuracy of the perturbation technique itself and the accuracy of the traveltime computation algorithm. The accuracy of the traveltime computation algorithm was discussed by Shin et al. (2002). To evaluate the accuracy of the perturbation technique, we simply compare the traveltimes and amplitudes computed by the perturbation technique with those computed using the target model as the initial reference model. In the following discussion, the "exact" solution means the traveltimes and amplitudes computed using the finite-element method where the target model is our initial reference model.

Figure 1 represents a simple three-layered earth model containing a $200 \mathrm{~m} \times 200 \mathrm{~m}$ rectangular perturbation centered at $(2000 \mathrm{~m}, 1500 \mathrm{~m})$ in the second layer. The velocities and densities of each layer are shown in Figure 1. Figure 2 shows the convergence rate of the L2-norm of the scattered wavefields when the velocity of the scattering region is perturbed to $2750 \mathrm{~m} / \mathrm{s}$, which is $10 \%$ higher than that of the reference medium. We note that the L2-norm converges rapidly within 3-4 iterations. In Figure 3, we plot the convergence rate of the L2-norm of the errors between the solutions at each iteration and the exact solutions. We can also have good estimates of the exact solutions with 3-4 iterations. In this study, we use the gradient of the L2-norm of the scattered wavefield as a stopping criterion. 


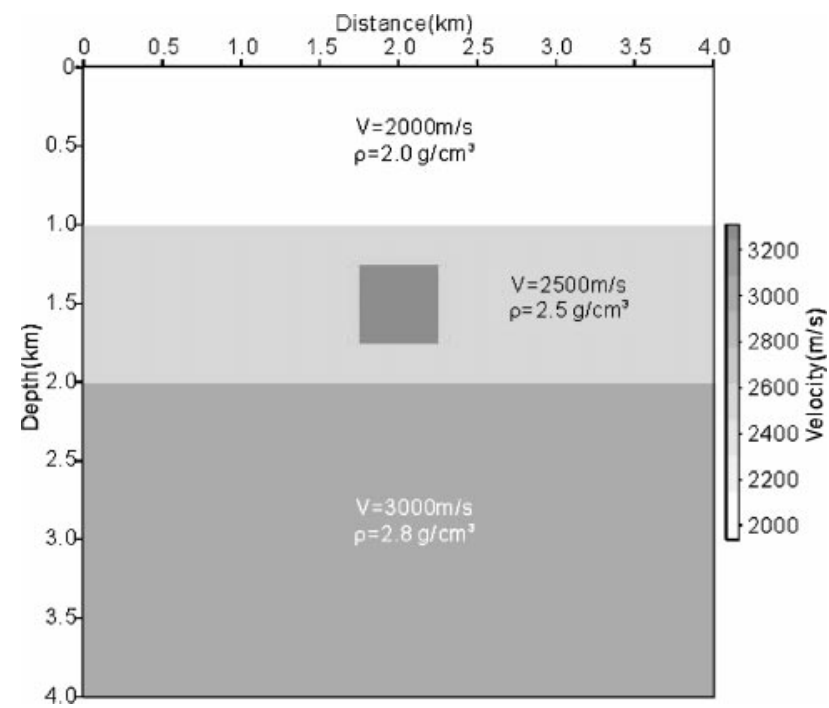

FIG. 1. A three-layered earth model containing a scatterer in the second layer.

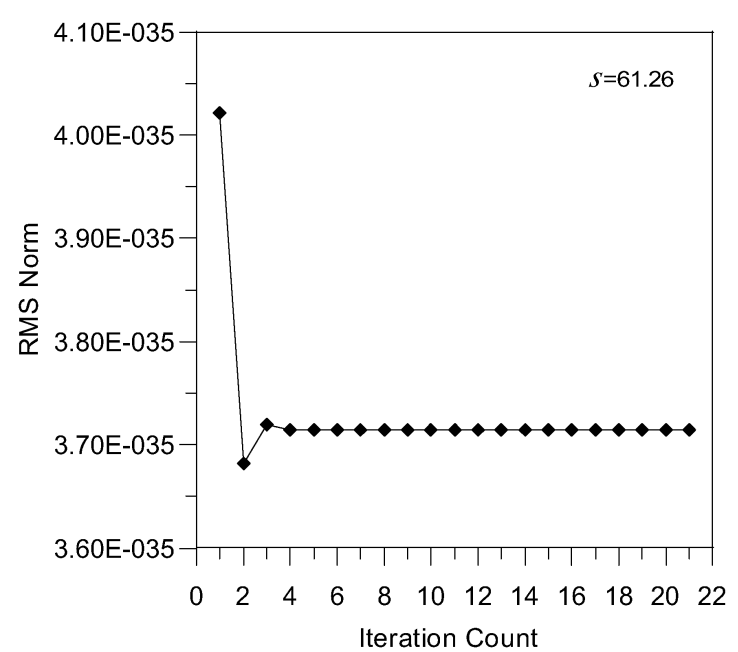

(a)

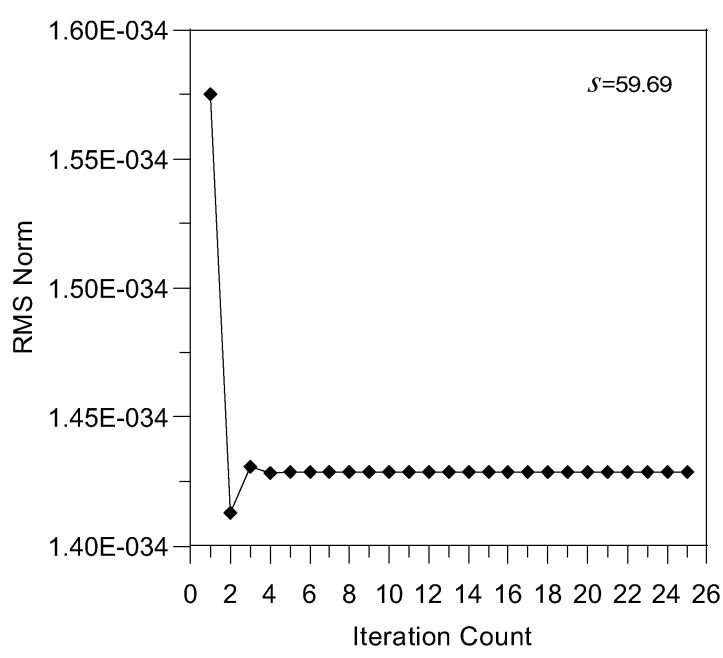

(b)

FIG. 2. Convergence of the L2-norm of the scattered wavefields as a function of the damping coefficient; $s$ is the Laplace frequency.
Shin et al.

In Figure 4, we analyze convergence and accuracy as a function of the percent change in velocity. For each percent change in velocity, we first obtain the solution using the previously mentioned stopping criterion, and then measure the relative L2-norm of the errors in the traveltimes and amplitudes. We note that the solutions rapidly converge with reasonable order of accuracy. Since the Born perturbation theory is based on a weak-scattering assumption, a somewhat large perturbation may break down the assumption. However, we note that the solutions converge within 10 iterations, even for a velocity perturbation of $30 \%$. In Figure $5 \mathrm{a}$, we display the traveltimes corresponding to the perturbed model (solid lines) and to the reference model (dashed lines) when the velocity of the scatterer is perturbed to $3250 \mathrm{~m} / \mathrm{s}$, which is $30 \%$ higher than the reference velocity of the second layer. As expected, the traveltime

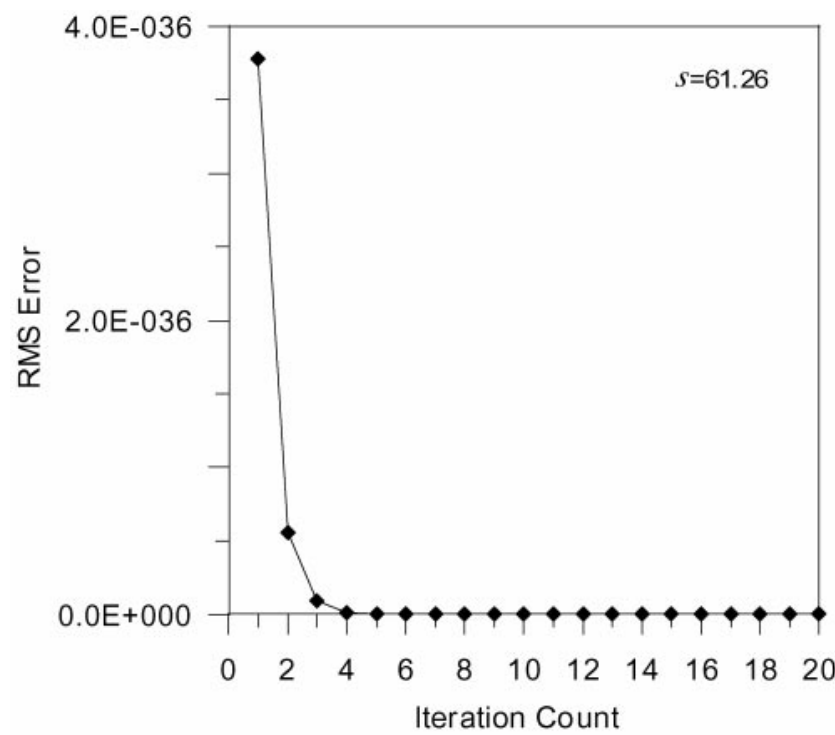

(a) Traveltime

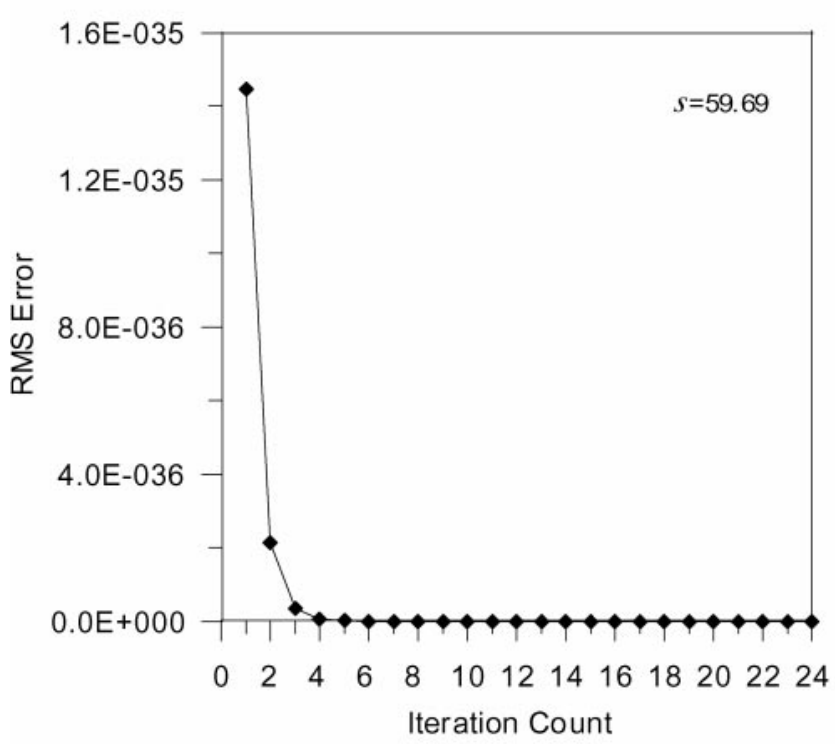

(b) Amplitude

FIG. 3. Convergence of the L2-norm of the errors between the solutions at each iteration and the exact solutions; $s$ is the Laplace frequency. 
contours are pushed out due to the high-velocity perturbation. The perturbed amplitude is shown in Figure 5b. We observe a complex pattern in the scattered zone due to the defocusing. The traveltime and amplitude errors are displayed in Figure 6. The traveltime errors are less than $0.001 \mathrm{~ms}$, whereas the amplitude error is less than $0.1 \%$. As previously mentioned, the given $30 \%$ deviation of velocity is somewhat larger than the amount that is generally considered in a weak-scattering assumption. Thus, we need more iterations to obtain an accurate solution than we would for a perturbation of only $10 \%$ or $20 \%$. For such a large perturbation, an alternative solution procedure is to recursively apply smaller iterative Born perturbation steps and bootstrap ourselves up to the final solution.

Figure 7 shows the traveltimes and amplitudes when the velocity of the scatterer is perturbed to $2000 \mathrm{~m} / \mathrm{s}$, which is $20 \%$ lower than the reference velocity of the second layer. The traveltime lags are due to a local low-velocity zone (Figure 7a), and the high amplitudes due to the trapped wave (Figure $7 \mathrm{~b}$ ) are easily identified. The traveltime and amplitude errors are shown in Figure 8. We note that for the same order of percent change of velocity perturbation, a low-velocity perturbation presents a more serious challenge. Nevertheless, the traveltimes and amplitudes agree well with the exact solution.

\section{Multiple scatterer model}

To further test our algorithm, we examine the more complex model shown in Figure 9, where our three-layered model contains three scatterers in the second layer. The velocities and densities of each layer are also shown in Figure 9. The upper left and upper right inhomogeneities are perturbed to $10 \%$ lower and $10 \%$ higher velocities, respectively, than that of the second layer. The lower inhomogeneity is perturbed to $20 \%$ higher than the background. In Figure 10a, we display the traveltimes of the reference model (dashed lines) and the perturbed model (solid lines), and in Figure $10 \mathrm{~b}$ we display

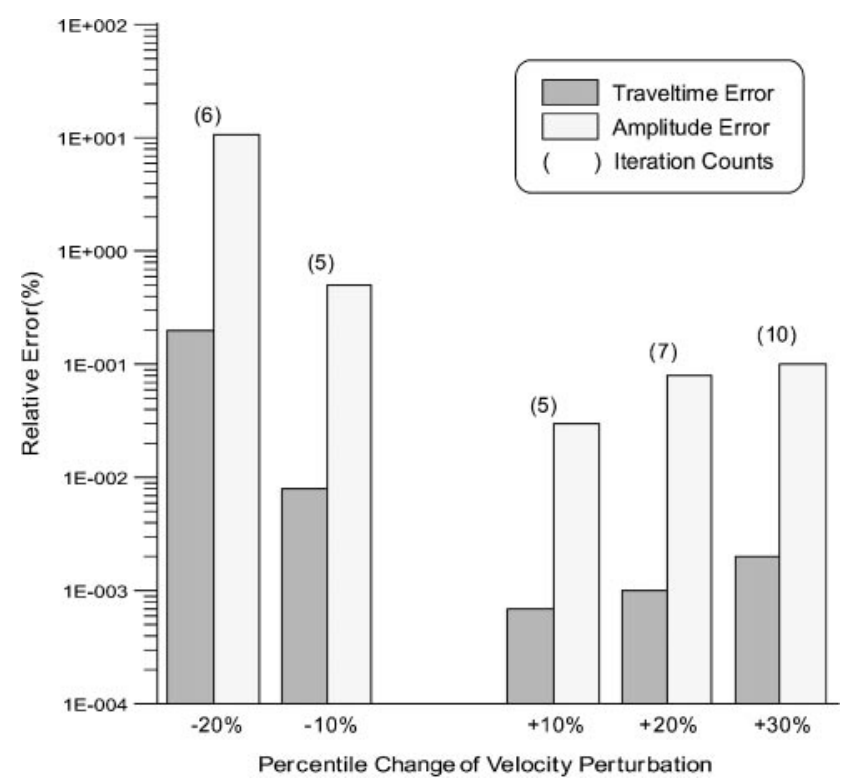

FIG. 4. Convergence and accuracy as a function of percent change in velocity. The numbers in the parenthesis denote the iteration counts where each solution is obtained. the perturbed amplitude. In Figure 11, we represent the errors between the exact solutions and the perturbed solutions after seven iterations. We note that the traveltime and amplitude errors are localized upon the highest perturbation region. Both traveltime and amplitude errors are compatible with the exact solutions. The maximum traveltime error is less than $0.001 \mathrm{~ms}$, whereas the amplitude error is less than $0.01 \%$. Thus, we find that the perturbation method works well even for a complex model having multiple scatterers.

\section{COMPUTATIONAL EFFICIENCY}

Unless we update the entire velocity model-and the zone to be updated is large in a prestack Kirchhoff migration-we compute traveltimes and amplitudes of some localized source points which are expected to influence the target zone.

We will use the simple, commonly understood band matrix solver (George and Liu, 1981), to illustrate the computational

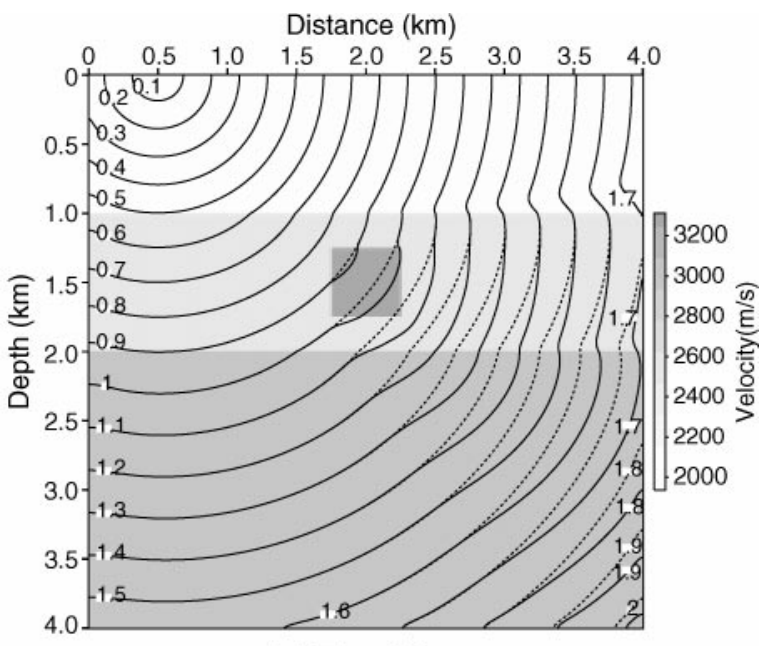

(a) Traveltime

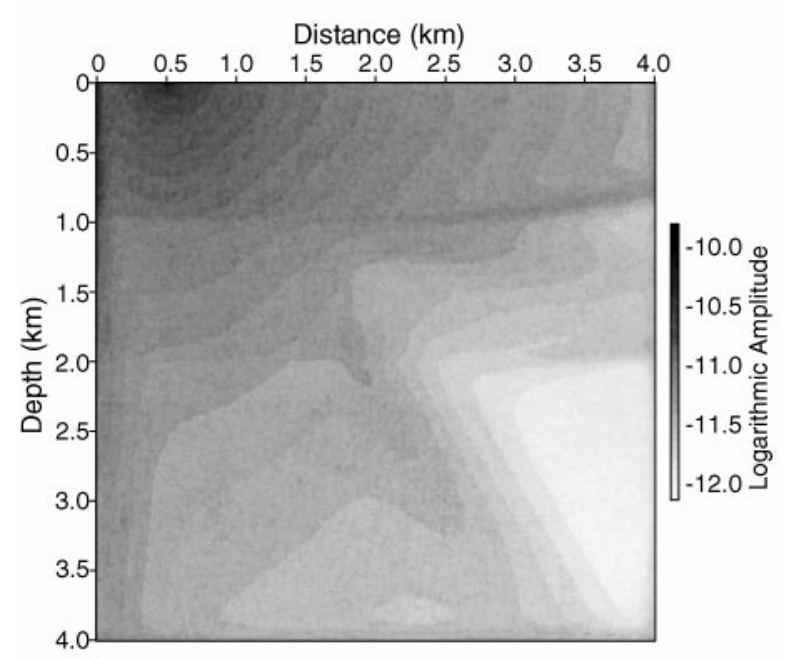

(b) Amplitude

FIG. 5. Traveltimes and amplitudes when the scatterer shown in Figure 1 is perturbed to $v=3250 \mathrm{~m} / \mathrm{s}$, which is $30 \%$ higher than the velocity of the reference medium. (a) The perturbed traveltimes (solid lines) and initial traveltimes (dashed lines), and (b) the perturbed amplitudes. 
efficiency of our perturbation technique with the direct solution of Shin et al.'s (2002) SWEET method. Greater savings are obtainable by using more efficient nested dissection or multifrontal solvers. In order to compute traveltimes and amplitudes for a given initial model, both the SWEET and perturbation methods begin by factoring the impedance matrix, $\mathbf{S}=\mathbf{S}_{\mathbf{L}} \mathbf{S}_{\mathbf{U}}$, arising from the initial model, where $\mathbf{S}_{\mathbf{L}}$ is a lower and $\mathbf{S}_{\mathbf{U}}$ an upper triangular matrix. To solve equation (2), we define an intermediate vector, $\mathbf{V}=\mathbf{S}_{\mathbf{U}} \mathbf{U}$, and then forward substitute $\mathbf{S}_{\mathbf{L}} \mathbf{V}=\mathbf{F}$ to obtain $\mathbf{V}$ from our sparse source distribution and backward substitute $\mathbf{S}_{\mathbf{U}} \mathbf{U}=\mathbf{V}$ to obtain our solution. Once we factor the impedance matrix in our perturbation technique given by equation (8), we repeat backward and forward substitutions originating from the updated velocity model and do not need to refactor the original impedance matrix. For most cases, the iteration number is no larger than 3 (e.g., Figures 2 and 3).

Suppose that the number of nodes in $x$ and $z$ are $N_{x}$ and $N_{z}$, respectively, for the model shown in Figure 12 . When we use

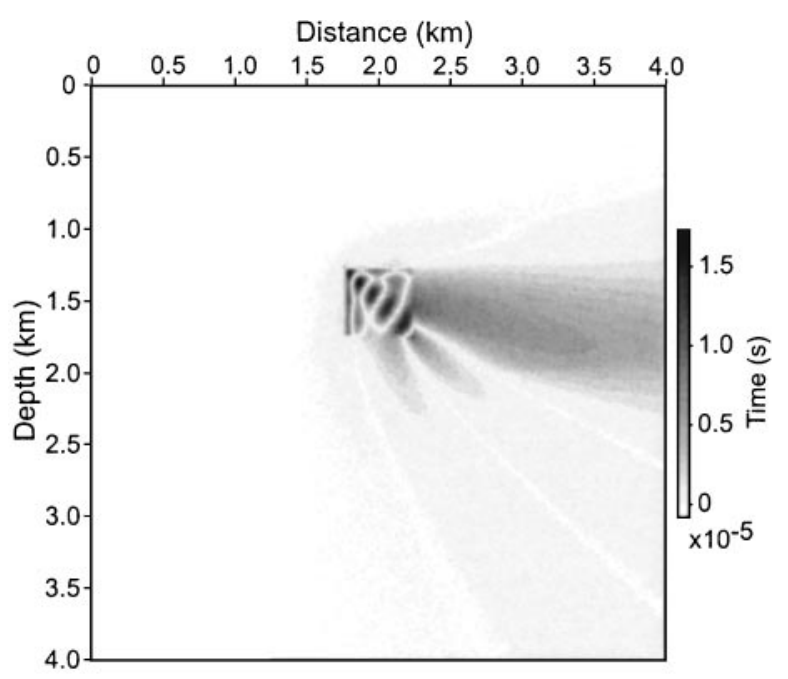

(a) Traveltime

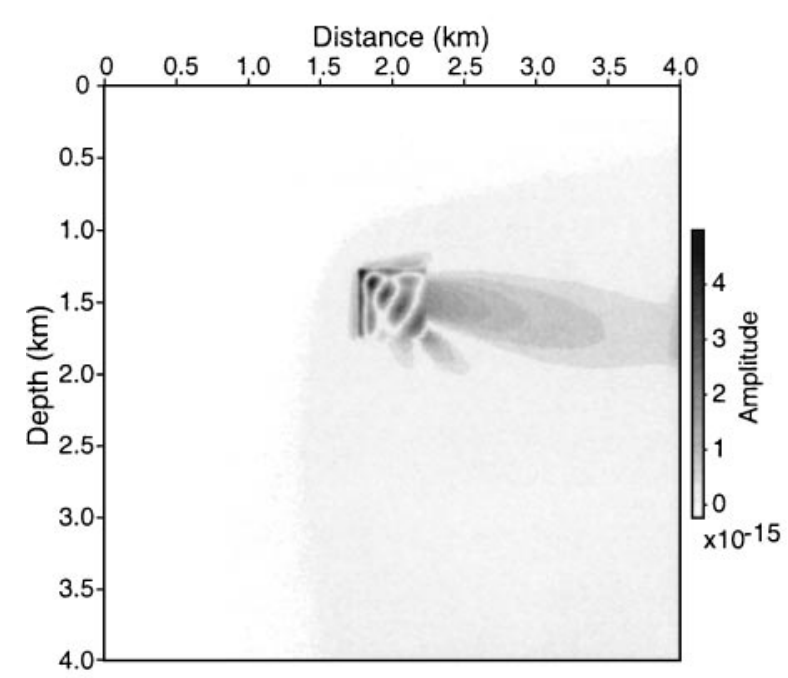

(b) Amplitude

FIG. 6. Traveltime (a) and amplitude (b) errors between the solutions shown in Figure 5 and the exact solution obtained by a direct finite-element solutions of the perturbed model. a band matrix solver, we first assemble the impedance matrix, $S$, resulting in a band width $N_{z}$ (for $N_{z}<N_{x}$ ). For a single frequency, the number of operations required for factoring this impedance matrix is then $\left(1 / 2 \times N_{x} \times N_{z}^{3}\right)$, while the number of operations required for backward and forward substitution is $\left(2 \times N_{x} \times N_{z}^{2}\right)$ (George and Liu, 1981). For both the SWEET and the perturbation methods, since we need to compute the wavefield and its derivative at a single frequency (Shin et al., 2002), we perform forward and backward substitution once more; that is, the number of operations required for backward and forward substitution is $\left(4 \times N_{x} \times N_{z}^{2}\right)$ for both methods. By numbering our nodes beginning at the surface and ending at depth level $N_{z}$, and noting that, as shown in Figure 12, if the subsurface perturbation influences $m$ source points, the total number of operations is $\left(1 / 2 \times N_{x} \times N_{z}^{3}+4 m \times N_{x} \times N_{z}^{2}\right)$ for the SWEET method, whereas the total number of operations for our perturbation method is $\left(4 n \times m \times N_{x} \times N_{z}^{2}\right)$, where $n$

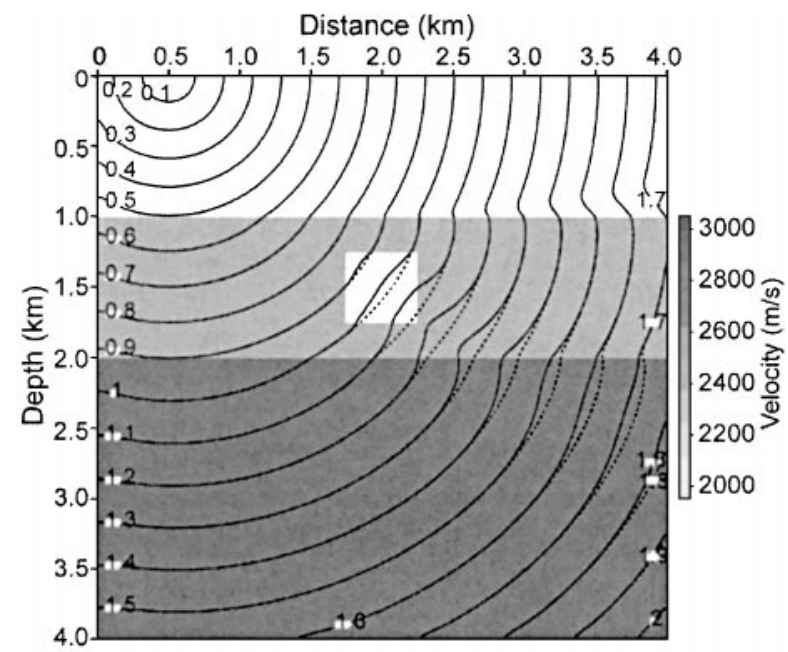

(a) Traveltime

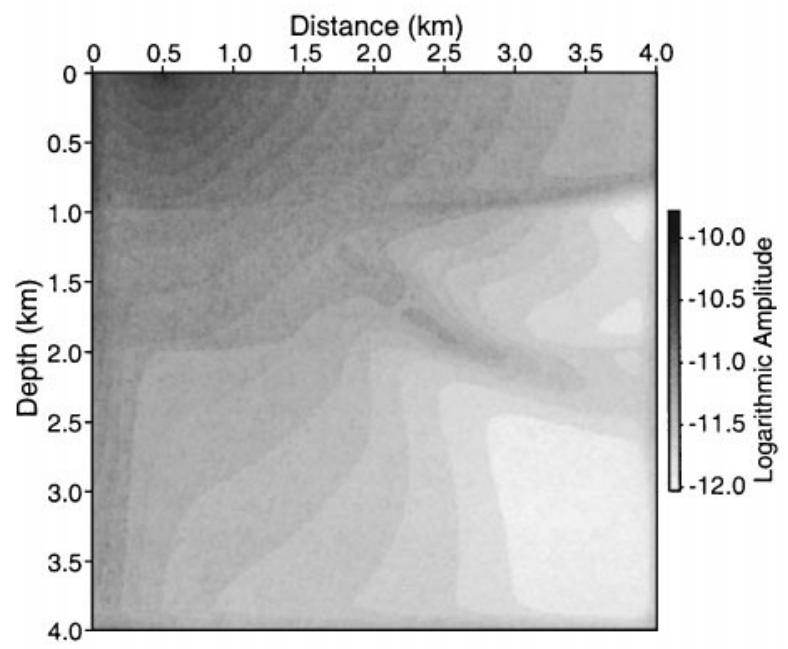

(b) Amplitude

FIG. 7. Traveltimes and amplitudes when the scatterer shown in Figure 1 is perturbed to $v=2000 \mathrm{~m} / \mathrm{s}$, whichis $20 \%$ lower than the velocity of the reference medium. (a) The perturbed traveltimes (solid lines) and initial traveltimes (dashed lines), and (b) the perturbed amplitudes. 


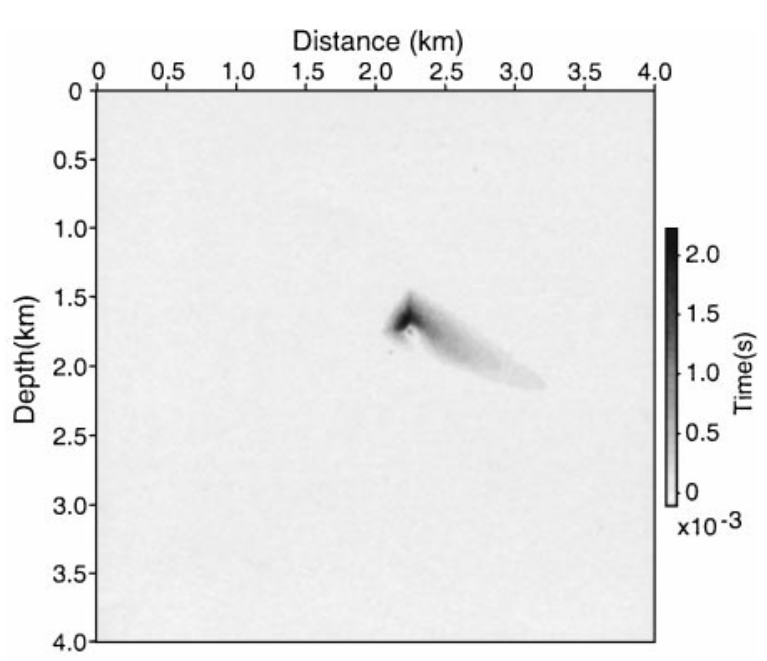

(a) Traveltime

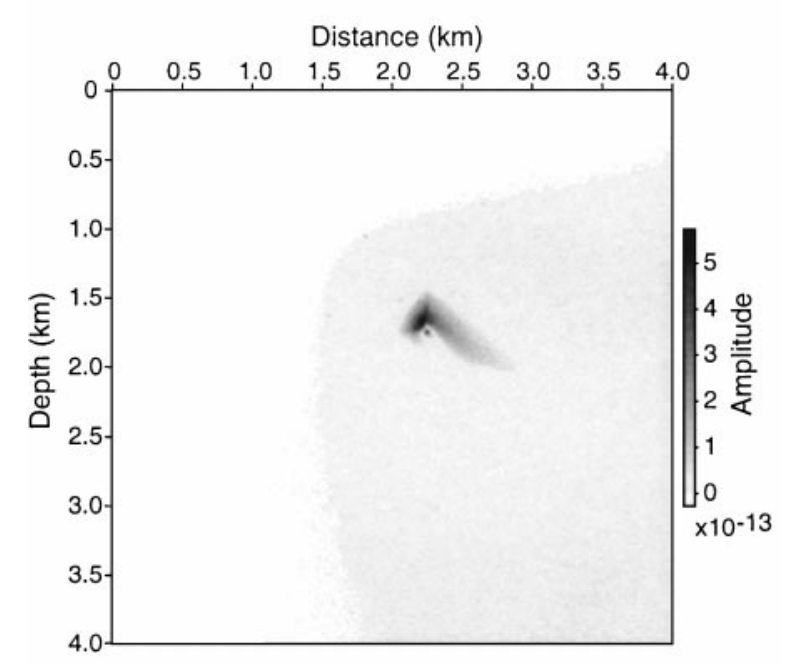

(b) Amplitude

FIG. 8. Traveltime (a) and amplitude (b) errors between the solutions shown in Figure 7 and the exact solutions.

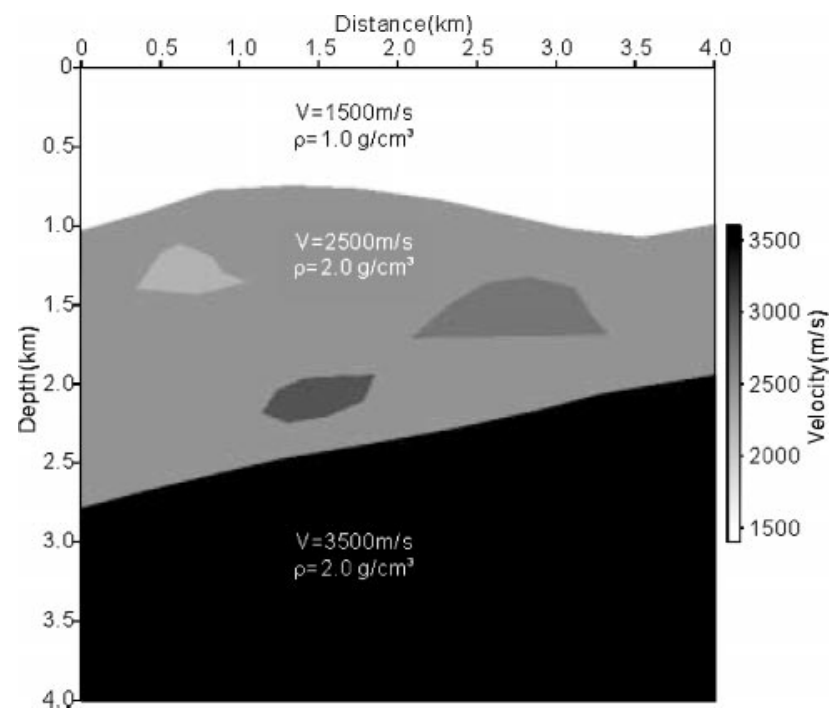

FIG. 9. A multiple-scatterer model having three scatterers in the second layer of a three-layered earth. is the number of iterations. The number-of-operations ratio of the direct method over the perturbation method is therefore $N_{z} / 8 m n+1 / n$. For $n=3$, we note that our perturbation method will be more efficient than the SWEET method when $N_{z}>16 \mathrm{~m}$. Values of $N_{z}=500$ are typical for seismic migration problems. In Table 1, we compare the number of operations in the SWEET method with that in our perturbation method.

\section{DISCUSSION AND CONCLUSIONS}

We have used an iterative Born perturbation theory applied to the damped wave equation algorithm (i.e., the Laplacetransformed wave equation algorithm) to compute the traveltimes of the acoustic wavefield for a suite of models. By implementing iterative Born perturbation theory and the damped wave equation algorithm, we can compute the traveltime and

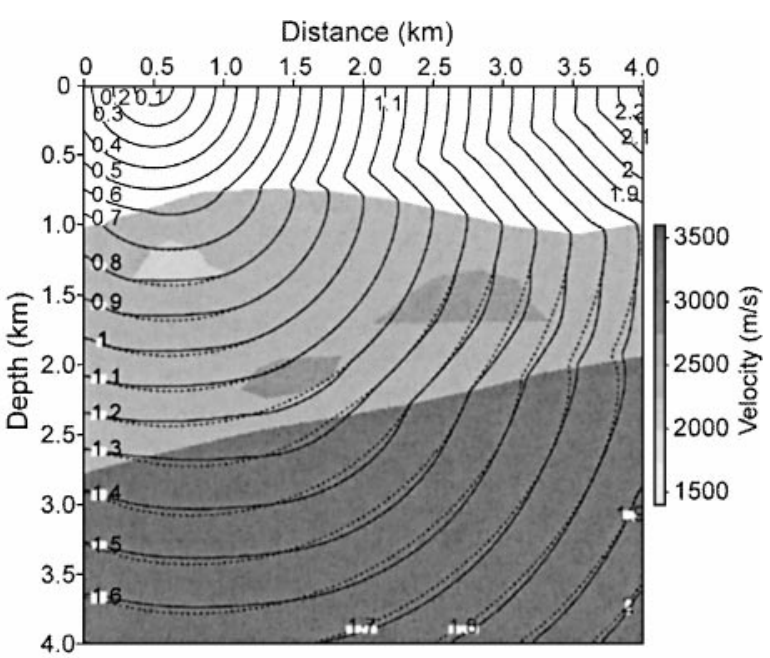

(a) Traveltime

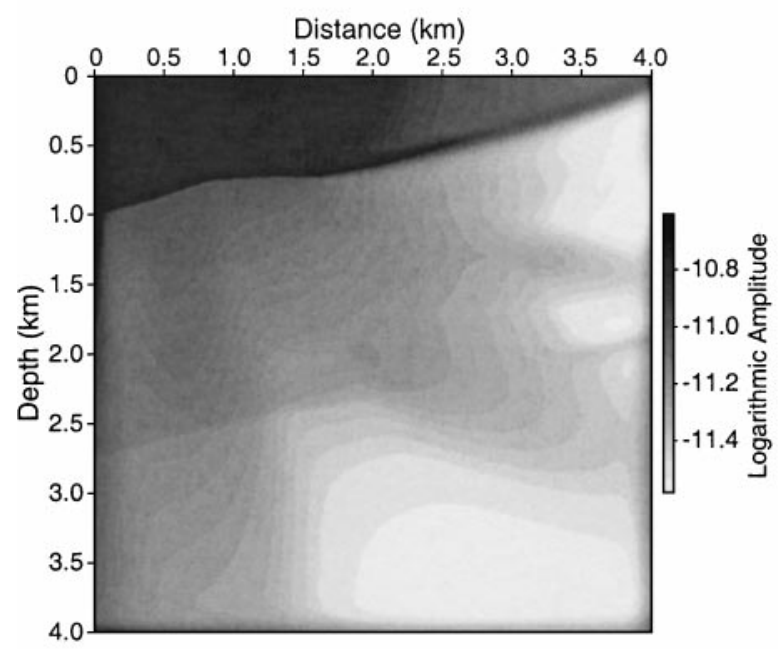

(b) Amplitude

FIG. 10. Traveltimes and amplitudes for the model shown in Figure 9. The upper left and the upper right scattering zones are perturbed to $10 \%$ lower $(2250 \mathrm{~m} / \mathrm{s})$ and $10 \%$ higher $(2750 \mathrm{~m} / \mathrm{s})$, respectively, than the reference velocity of the second layer. The lower scattering zone is perturbed to $20 \%$ higher velocity $(3000 \mathrm{~m} / \mathrm{s})$. (a) The perturbed traveltimes (solid lines) and initial traveltimes (dashed lines). (b) The perturbed amplitudes. 
amplitude with reasonable efficiency. We obtain stable solutions for models having velocity perturbations up to $30 \%$ greater than the reference velocity. Our algorithm produces accurate results even when we encounter either discontinuous traveltime gradients or shadow zones, where the usual methods based on the high-frequency ray approximation often fail.

We feel that our perturbation technique can make iterative velocity model refinement in migration more affordable. This method will be very cost effective when a relatively small num-

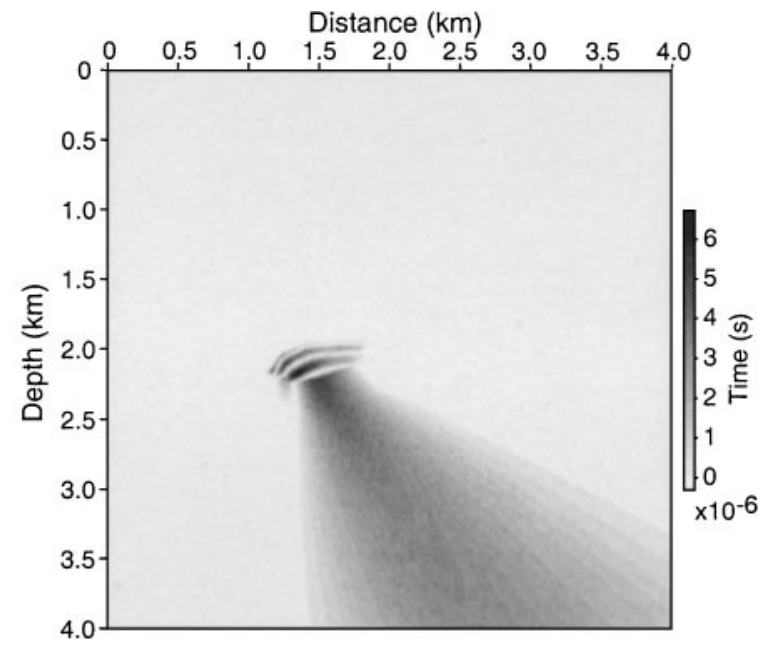

(a) Traveltime

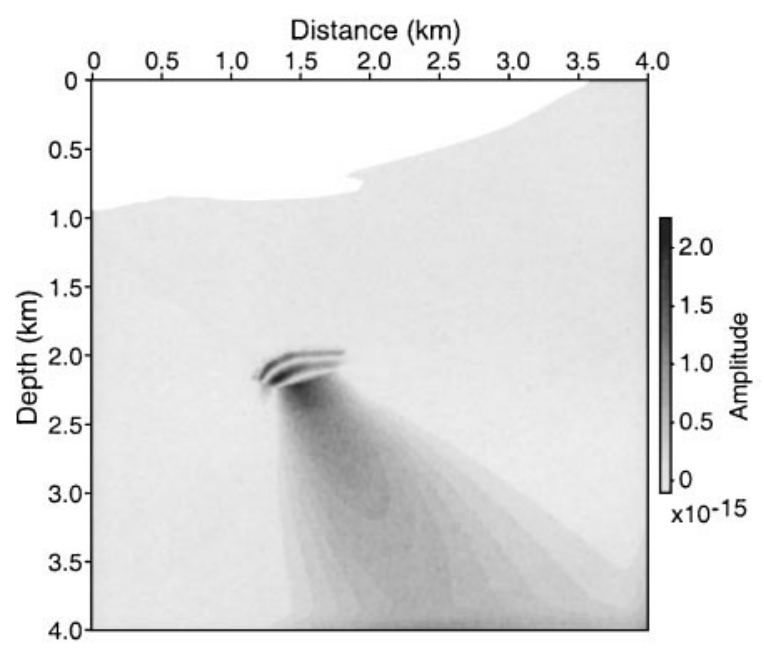

(b) Amplitude

FIG. 11. Traveltime (a) and amplitude (b) errors between the solutions shown in Figure 10 and the exact solutions. ber of sources or receivers (where we could use reciprocity) are perturbed as encountered in reverse vertical-seismic-profiling and ocean-bottom-cable experiments. Unfortunately, the cost increases linearly with the number of sources effected by the perturbation. We find that for production marine-streamer and land-surface acquisition geometries where all shot gathers are perturbed, a simple resolution of the problem using modern sparse matrix solvers such as nested dissection provides a more efficient means of updating the traveltimes than the perturbation method described in this paper.

Although we did not show examples in this paper, the issue of anisotropy in both acoustic and elastic problems can easily be incorporated using perturbation techniques. Moreover, all the amplitude effects due to variation of density and Lamé constants, and attenuation, can be easily addressed.

\section{ACKNOWLEDGMENT}

This work was financially supported by National Research Laboratory Project of the Korea Ministry of Science and Technology, Brain Korea 21 project of the Korea Ministry of Education, grant No. R05-2000-00003 from the Basic Research Program of the Korea Science \& Engineering Foundation, and grant No. PM10300 from Korea Ocean Research \& Development Institute.

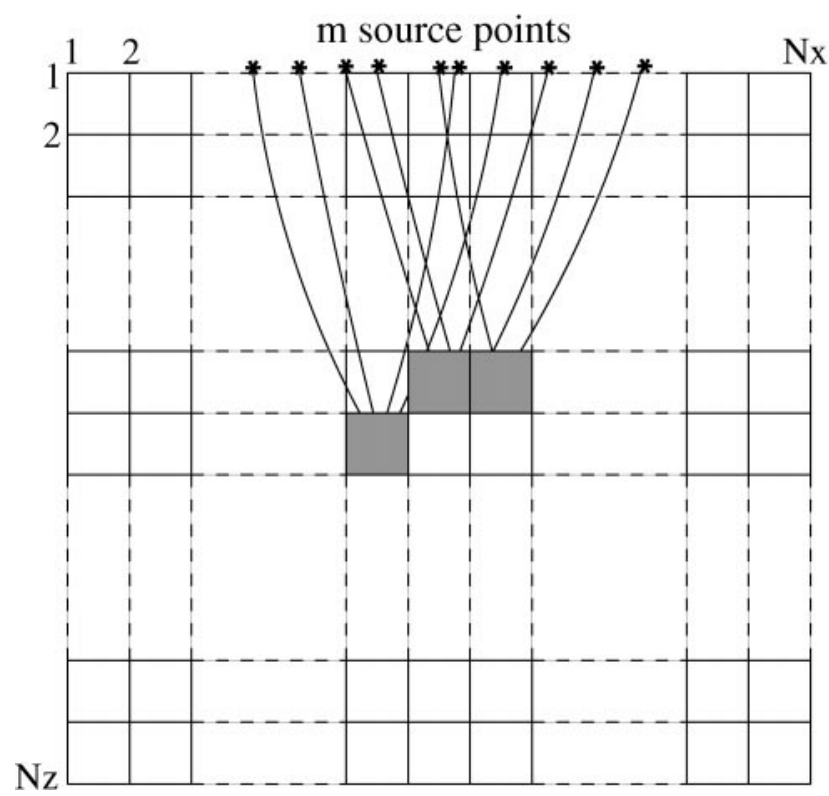

FIG. 12. A simple perturbed model. Target zones are shaded, and local source points are denoted by*

Table 1. Comparison of the number of operations in the SWEET method and our perturbation method required to calculate traveltimes and amplitudes for $m$ shot points.

\begin{tabular}{lcc}
\hline & SWEET method & Perturbation method \\
\hline $\begin{array}{l}\text { Number of operations for factoring matrix } \\
\text { Number of operations for forward and }\end{array} \quad 1 / 2 \times N_{x} \times N_{z}^{3}$ & - \\
$\quad$ backward substitution & $4 m \times N_{x} \times N_{z}^{2}$ & $4 n \times m \times N_{x} \times N_{z}^{2}$ \\
Total number of operations & $1 / 2 \times N_{x} \times N_{z}^{3}+4 m \times N_{x} \times N_{z}^{2}$ & $4 n \times m \times N_{x} \times N_{z}^{2}$ \\
Total number of operations for $n=3$ & $1 / 2 \times N_{x} \times N_{z}^{3}+4 m \times N_{x} \times N_{z}^{2}$ & $12 m \times N_{x} \times N_{z}^{2}$ \\
\hline
\end{tabular}




\section{REFERENCES}

Ettrich, N., and Gajewski, D., 1998, Traveltime computation by perturbation with FD-eikonal solver in isotropic and weakly anisotropic media: Geophysics, 63, 1066-1078.

George, A., and Liu, J. W., 1981, Computer solution of large sparse positive definite systems: Prentice-Hall, Inc.

Hohmann, G. W., 1990, Three-dimensional IP models, in Fink, J. B., McAlister, E. O., Sternberg, B. B., Wiederwilt, G. W., and Ward, S. H., Eds., Induced Polarization-Applications and case histories: Soc. Expl. Geophys., 150-178.

Marfurt, K. J., 1984, Accuracy of finite-difference and finite-element modeling of the scalar and elastic wave equations: Geophysics, 49, 533-549.

Marfurt, K. J., and Shin, C., 1989, The future of iterative modeling in geophysical exploration, in Eisner, E., Ed., Handbook of geophysica exploration: I-Seismic Exploration: Pergamon Press, 203-228.

Podvin, P., and Lecomte, I., 1991, Finite-difference computation of traveltimes in very contrasted velocity models: A massively parallel approach and its associated tools: Geophys. J. Internat. 105, 271-284.
Qin, F., Luo, Y., Olsen, K. B., Cai, W., and Schuster, G. T., 1992, Finitedifference solution of the eikonal equation along expanding wave fronts: Geophysics, 57, 478-487.

Shin, C., Min, D.-J., Marfurt, K. J., Lim, H. Y., Yang D., Cha, Y., Ko, S., Yoon, K., Ha, T., and Hong, S., 2002, Traveltime and amplitude calculations using damped wave equation; Geophysics, 67, 16371647, this issue.

Stolt, R. H., and Weglein, A. B., 1985, Migration and inversion of seismic data: Geophysics, 50, 2458-2472.

Tarantola, A., 1987, Inverse problem theory: Elsevier Science Publ. Co., Inc.

van Trier, J., and Symes, W. W., 1991, Upwind finite-difference computation of traveltimes: Geophysics, 56, 812-821.

Vidale, 1988, Finite-difference calculation of traveltimes: Bull. Seis Soc. Am., 78, 2062-2076.

Zienkiewicz, O. C., 1977, Finite-element method: McGraw-Hill Book Co. Ltd.

Zhu, J., and Lines, L. R., 1998, Comparison of Kirchhoff and reversetime migration methods with applications to prestack depth imaging of complex structures: Geophysics, $\mathbf{6 3}, 1166-1176$. 